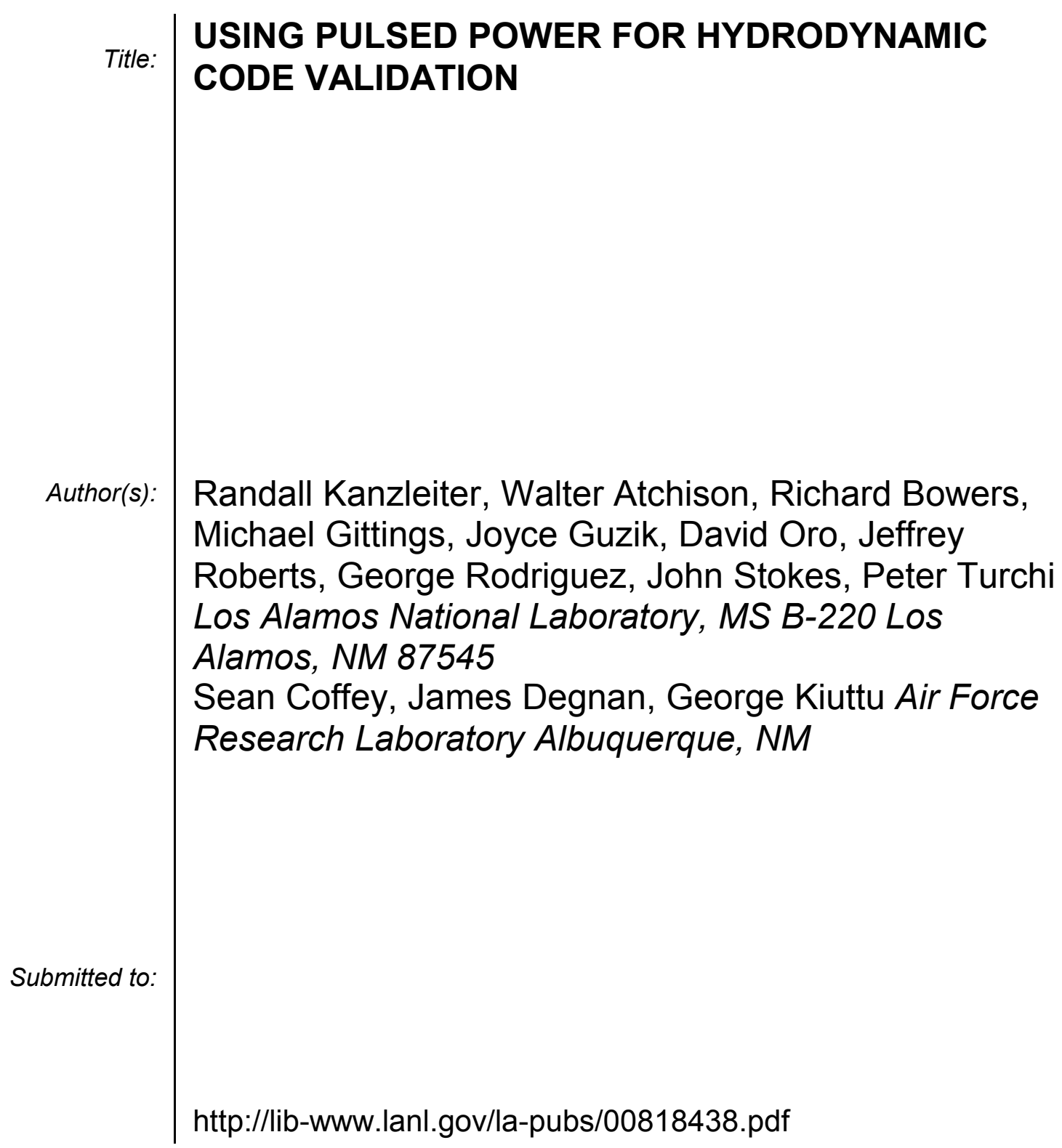




\title{
USING PULSED POWER FOR HYDRODYNAMIC CODE VALIDATION
}

\author{
Randall Kanzleiter, Walter Atchison, Richard Bowers, Michael Gittings, Joyce Guzik, David \\ Oro, Jeffrey Roberts, George Rodriguez, John Stokes, Peter Turchi \\ Los Alamos National Laboratory, MS B-220 \\ Los Alamos, NM 87545
}

\author{
Sean Coffey, James Degnan, George Kiuttu \\ Air Force Research Laboratory \\ Albuquerque, NM 87117
}

\section{Abstract}

As part of ongoing hydrodynamic code validation efforts, a series of Near Term Liner Experiments (NTLX) was designed for the Shiva Star capacitor bank at the Air Force Research Laboratory (AFRL). A cylindrical aluminum liner that is magnetically imploded onto a central target by self-induced radial Lorentz forces drove the experiments. The behavior of the target was simulated using the adaptive mesh refinement (AMR) Eulerian hydrodynamics code RAGE in 2- and 3-D. Onedimensional simulations of the liner driver utilizing the RAVEN MHD code were used to predict the liner density and temperature profiles as well as the velocity at impact time. At liner/target impact, a convergent shock is generated that drives subsequent hydrodynamics experiments. In concentric targets, cylindrically symmetric shocks will converge on axis. This characterizes the symmetry of the liner driver. By shifting the target center away from the liner symmetry axis, material dependencies in the shock propagation velocity generate off-center shock convergence. Both codes show excellent agreement over the majority of the shock and interface trajectories. However, a small but significant discrepancy between codes does occur during the last few millimeters of run in when convergence effects are greatest. Comparisons with experimental data show similar shock velocities being measured. However, the simulated shock arrives at a given trajectory location nearly $100 \mathrm{~ns}$ earlier than experimentally measured.

\section{INTRODUCTION}

With the increased reliance on numerical simulation for problems in complex geometries, questions arise as to the accuracy of the results produced. Concerns of this nature emphasize the importance of validating that computer codes can successfully predict material behavior in relatively simple geometries let alone under more complex scenarios. The purpose of this effort was to design a pulsed power experiment to obtain high quality data of sufficient accuracy to characterize hydrodynamic phenomena in convergent geometries. As a preliminary step, a series of Near Term Liner Experiments (NTLX) was designed and fielded on the Shiva Star Pulsed Power Machine at AFRL.

\section{COMPUTATIONAL TOOLS}

Experiment design and pre-shot calculations were performed using the adaptive mesh refinement (AMR) Eulerian hydrodynamics code RAGE [1]. RAGE lacks the electromagnetic effects describing pulsed power magnetic drives such as those produced at Shiva Star, so the one-dimensional Lagrangian code RAVEN [2], [3] was used to model MHD effects and to set the initial liner/target interaction parameters.

The RAGE code is a one-, two- or three-dimensional, multi-material Eulerian hydro code utilizing adaptive mesh refining (AMR) and SESAME [4] equation of state data. Code features and assumptions employed within RAGE include: 1-D cylindrical coordinate systems, 2-D cartesian and cylindrical coordinate systems, 3-D cartesian coordinate system, continuous adaptive mesh refinement on a cell-by-cell basis, arbitrary number of materials per cell, pressure and temperature equilibrium for all materials in a cell, higher order piecewise linear Godunov numerical method to simulate shocks, exact conservation of mass, momentum and total energy as well as dynamic program memory allocation.

RAVEN is a 1-D Langrangian MHD code utilizing artificial viscous stresses to represent shocks and SESAME equation-of-state data to model the liner driver and subsequent shock evolution through the NTLX target. The pulsed power drive for the NTLX experiments is represented through a self-consistent circuit model employing a lumped RLC circuit. This circuit model allows for self-consistent calculations of coupling between the pulsed power system and the imploding liner driver. RAVEN includes a Steinberg-Guinan [5] strength model along with a tabular melt model for the aluminum liner driver. It uses SESAME resistivity data to complete the circuit model and determine the liner state (density, 
temperature and velocity) at impact. These state parameters are then used as initial conditions for the multidimensional RAGE hydro calculations of the target.

\section{EXPERIMENTAL ARRANGEMENT}

The NTLX experimental series employed a previously characterized cylindrical aluminum liner driver that is magnetically imploded onto a central target. Following the onset of current flow, the self-induced radial Lorentz force accelerates the $1 \mathrm{~mm}$ thick aluminum liner to a radial velocity of $6.25 \mathrm{~mm} / \mu \mathrm{s}$ at a target radius of $2 \mathrm{~cm}$. The convergent shock generated at liner/target impact drives subsequent hydrodynamic experiments to characterize shock behavior in both symmetric and asymmetric geometries shown respectively in fig. 1a and $1 \mathrm{~b}$.

The NTLX target was designed with two material layers to investigate hydrodynamic phenomena in convergent geometry. The outer target region consists of a Sn casing used to isolate hydrodynamic issues and shock convergence effects from the MHD drive used at Shiva Star. For shock pressures greater than approximately $300 \mathrm{kbar}$, the outer Sn target melts. This removes complications due to material strength from comparisons of experimental data with simulations. Liner/target impacts in the NTLX series generate shock pressures greater than $1 \mathrm{Mbar}$, well above the shock pressure needed for the solid/liquid phase transition. The inner target consisted of a Lucite core to facilitate visualization of both the transmitted shock and the $\mathrm{Sn} /$ Lucite material interface.

Visualization of the shock/interface behavior and timing of the liner/target impact were accomplished by axial radiography and a series of B-dot probes respectively. The B-dot probes measured highly symmetric liner impacts with symmetries, $\Delta \mathrm{r} / \mathrm{r}$, between
97.5 and $99 \%$ at a radius of $2 \mathrm{~cm}$. Radiographically, resolutions on the order of $0.09 \mathrm{~mm}$ were routinely obtained for both the shock and interface locations.

\section{RESULTS}

Data obtained from the symmetric targets (NTLX-1 \& NTLX-3) allows a direct comparison between the RAVEN calculations including the MHD drive and RAGE 2- and 3-D hydrodynamic runs. Although based on different solution techniques, both RAGE and RAVEN results agree very well over most of the shock trajectory as seen in fig. 2. Only during the last few millimeters where convergence effects dominate do the codes diverge significantly. Comparison of the simulated and radiographed data shows a generally good agreement, with the simulated shock slightly leading the radiographic data over the entire implosion.

Besides shock trajectory information, radiographic analysis of the $\mathrm{Sn} /$ Lucite interface produces data on material behavior under strong shock loading. Figure 3 illustrates this point as RAVEN simulations including a Steinberg-Guinan strength model and RAGE simulations without strength produce identical results for the time dependent $\mathrm{Sn} /$ Lucite interface position. The late time radiographic data in fig.3, after $3.5 \mu \mathrm{s}$, was serendipitously obtained due to the initial Shiva Star bank voltage being under estimated by $2 \mathrm{kV}$. The increased bank voltage resulted in a liner/target impact $2 \mu$ s earlier then predicted.

The divergence between the simulated and radiographed interface at $3 \mu$ s occurs due to visualization limitations of the axial radiography. The edge of the converging shock weakens and slows due to disturbances near the edge of the liner impact region. The central portion of the converging shock is the strongest and thus reflects on axis first and interacts a second time with the

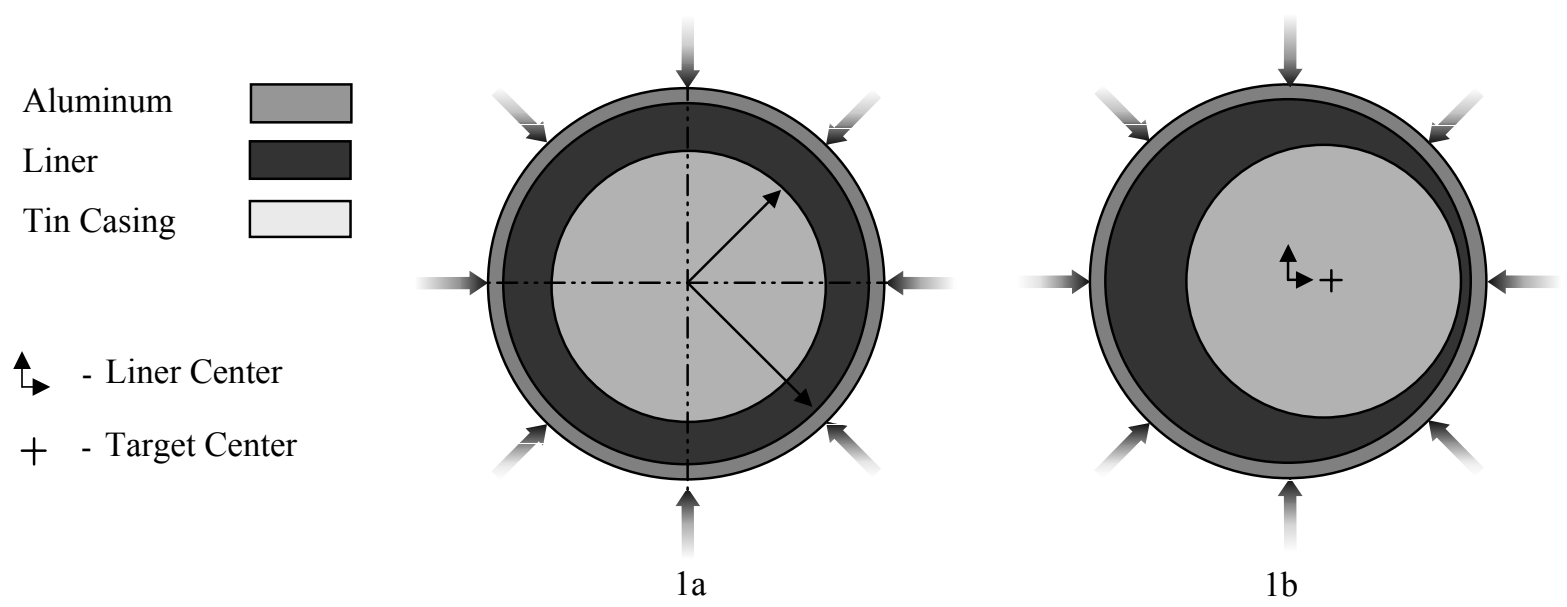

Figure 1. Target arrangements used during the NTLX liner implosion experiments. 1a) symmetric target and $1 \mathrm{~b}$ ) asymmetric target configurations. Shown are the aluminum liner driver, outer Sn casing and inner Lucite core. 
$\mathrm{Sn} /$ Lucite interface. Since the simulations focus on the target mid-plane, this causes the expansion of the simulated interface as seen in fig. 3 The radiography images the upper edge of the $\mathrm{Sn} /$ Lucite boundary and therefore does not see the reflected shock until much later in time.

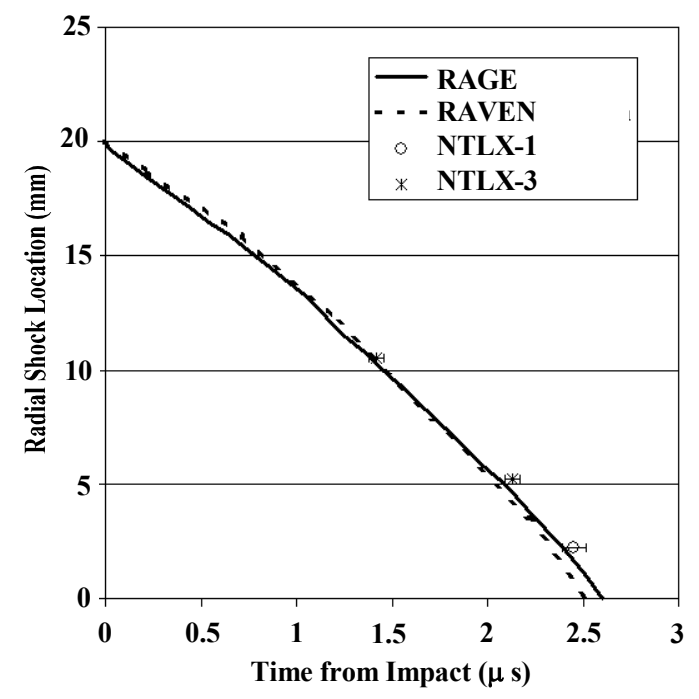

Figure 2. A high level of agreement exists between measured and simulated shock trajectories. Comparisons between RAGE and RAVEN show the greatest discrepancy near axis where convergence effects dominate.

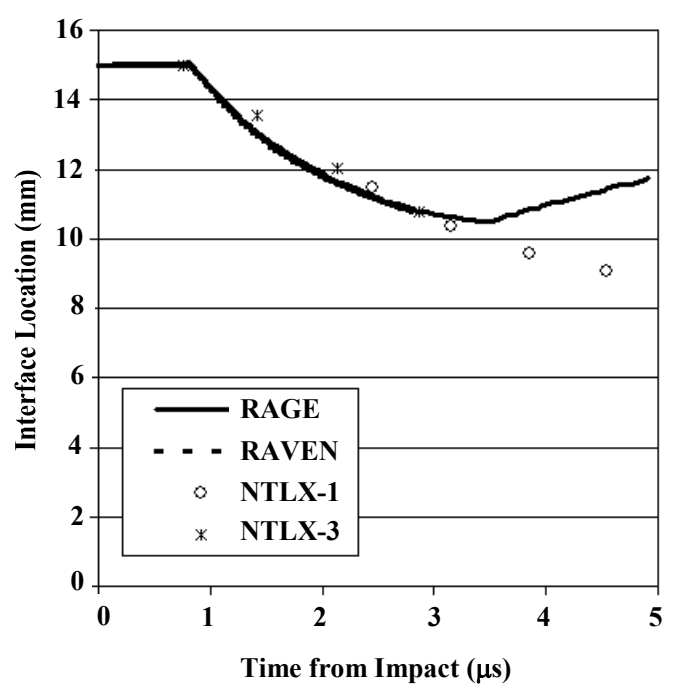

Figure 3. Both codes predict identical behavior of the material interface with and without strength effects. The radiographed interface exhibits a slightly lower compression rate than that calculated. The divergence at $3.5 \mu$ s is due to calculated shock interface interactions that are not experimentally observable.
In addition to the symmetric target shots, experiments NTLX-2 \& NTLX-4 examined converging shocks in an asymmetric geometry. The target shown in fig. $1 \mathrm{~b}$ shows an inner Lucite target that is shifted by $4 \mathrm{~mm}$ from the liner driver axis to produce off-center shock convergence. Figure 4 shows 2-D simulated and radiographed shock boundaries for the asymmetric target overlaid on a static radiographic image of the pre-shot target. As with the symmetric target, the simulated shocks were found to consistently lead the measured shock location by approximately $100 \mathrm{~ns}$. Timing discrepancies of this order are on the verge of what can be distinguished experimentally. Liner/target collisions are not precisely uniform with a collision as measured by B-dot probes taking between $26 \mathrm{~ns}$ and $123 \mathrm{~ns}$ to complete. Interpretation of the B-dot signal itself relies on RAVEN simulations to characterize the delay between the actual impact and generation of a probe signal. Imprecision in determining the experimental impact time leads to relative timing discrepancies on a 100 ns time scale. The simulated shocks in fig. 4 are adjusted by $104 \mathrm{~ns}$ to correct for this relative timing discrepancy and compare shock geometries. With the slight adjustment in timing between simulation and experiment, excellent agreement is obtained in the shock shapes from radiographic frames 1 and 3 from NTLX-2. The discrepancy observed in the middle frame is believed to be due to camera distortion since it is not observed in either the preceding or following frame.

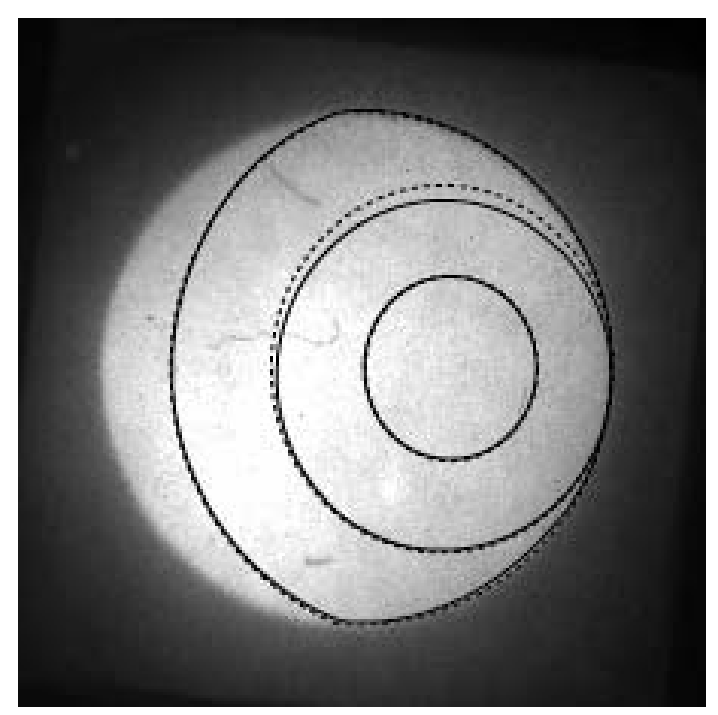

Figure 4. Simulated (--------) and radiographed (shock trajectories are compared in 2D after applying a relative timing correction of $104 \mathrm{~ns}$ to the simulated results. 


\section{CONCLUSIONS}

With the increased interest in using numerical simulation as a predictive tool, it is necessary to ensure that there exists a sufficient level of confidence in general use codes. These codes must be capable of simulating relatively simple experiments as well as more complex geometries. The current efforts focused on the design and comparison of data from the NTLX experimental series with the RAVEN and RAGE codes at Los Alamos National Laboratory. Both codes produce equivalent results for the shock trajectory in a symmetric twomaterial target except in the near-axis region where convergence effects dominate. Simulations of the $\mathrm{Sn} /$ Lucite interface produce identical results indicating that material strength effects are not significant for strong shock loading in Sn.

Detailed comparisons with experimental observations indicate that the simulations are predicting the correct shock velocity, but the calculated shock consistently arrives earlier at any given radial location. This points towards differences in the absolute timing on the order of $100 \mathrm{~ns}$ in the liner/target impact that generates the converging shock. Computationally, the impact is a precisely defined occurrence. Experimentally, generation of the converging shock and timing of the impact is complicated by azimuthal variations in the impact timing and delays in generation of the B-dot data signaling impact. Precise timing comparisons of shock data between simulations and experiments will inherently be subject to relative timing differences on the $100 \mathrm{~ns}$ level. This discrepancy may be reduced to the $10 \mathrm{~ns}$ level with precision liner implosions and better understanding of the B-dot signal generation.

\section{REFERENCES}

[1] R.M. Baltrusaitis, M.L. Gittings, R.P. Weaver, R.F. Benjamin and J.M. Budzinski, "Simulation of shock-generated instabilities," Phys. Fluids, vol. 8, pp. 2471-2483, Sept. 1996.

[2] T.A. Oliphant, "RAVEN Physics Manual," Los Alamos National Laboratory, Los Alamos, NM, Tech. Rep. LA-8802-M, June 1981.

[3] T.A. Oliphant and K.H. Witte, "RAVEN," Los Alamos National Laboratory, Los Alamos, NM, Tech. Rept. LA-10826, Jan. 1987.

[4] S.P. Lyon and J.D. Johnson, "SESAME: the Los Alamos National Laboratory Equation of State Database," Los Alamos National Laboratory, Los Alamos, NM, Tech. Rept., LA-UR-92-3407, 1992.

[5] D.J. Steinberg, S.G. Cochran and M.W. Guinan, "A constitutive model for metals applicable at high-strain rate," J. Appl. Phys., vol. 51, pp. 1498-1504, Mar. 1980. 